\title{
Factors Affecting the Temperature Increasing Rate in Arc-shaped Bamboo Pieces during High-frequency Heating
}

\author{
Feihu Chang, ${ }^{\text {a,b }}$ Yanhe Liu, ${ }^{\text {a,b }}$ Bin Zhang, ${ }^{\text {a,b }}$ Wansi Fu, ${ }^{\mathrm{b}}$ Pengfei Jiang, ${ }^{\mathrm{a}, \mathrm{b}}$ and \\ Jian-bo Zhou ${ }^{\text {a,b,* } *}$
}

\begin{abstract}
In the process of applying the high-frequency heating technology to bamboo heat treatment, controlling the material temperature has a great influence on the quality of bamboo forming. Therefore, research on the heat transfer mechanism of high-frequency heating of arc-shaped bamboo pieces is of great importance. In this paper, the influence of different moisture content, chord length, and plate voltage on the heating rate of arc-shaped bamboo pieces under high-frequency electric field were studied. The moisture content of bamboo had the most remarkable effect on the heating rate. With increased moisture content, the temperature rose faster. The selection of the plate voltage had an obvious influence on the heating. If the voltage was low, the heating rate was too slow, the heating time was long, or the voltage was high, it was easy to cause electric field breakdown and damage the bamboo pieces. As the chord length decreased, the heating rate gradually increased. When the radian of the arc-shaped bamboo pieces could be ignored, the heating rate was the fastest. The results showed that under certain conditions, the arc-shaped bamboo pieces showed a good heat treatment effect in a high-frequency electric field.
\end{abstract}

Keywords: High-frequency heating; Arc-shaped bamboo pieces; Heating rate; Influence

Contact information: a: Institute of New Forest Technology, Chinese Academy of Forestry, Beijing, China, 100091; b: Beijing Forestry Machinery Research Institute of State Forestry and Grassland Administration, Beijing, China, 100029; *Corresponding author: zhoujianbol@126.com

\section{INTRODUCTION}

With the increasing shortage of wood resources, the demand for bamboo that can replace wood resources is increasing (Guo et al. 2009; Fan 2014). High-quality, fast, and energy-saving heating technology is increasingly important and has become an urgent problem to be solved (Lv et al. 2015; Chang et al. 2018). High-frequency heating is an ideal method for gluing and drying bamboo. It has the characteristics of high cleanliness, strong effectiveness, high efficiency, and so on. Therefore, the use of high-frequency heating has gradually attracted the attention of scholars at home and abroad. Bamboo temperature is an important control parameter in the high-frequency heating process. The temperature distribution and heating trend of bamboo not only affect the heating speed of bamboo, but also affect the processing quality of bamboo (Pereira et al. 2004; Chai et al. 2018). It is of great significance to deeply study the heat transfer mechanism of highfrequency heating of bamboo and accurately understand the temperature change law of bamboo during the heating process.

The arc-shaped original bamboo pieces are mainly used in original state arc reorganization of bamboo. Original state arc reorganization of bamboo is the a new efficient utilization way in the field of bamboo processing which bionics is used, which 
keeps the natural bamboo arc structure, improve the utilization rate of bamboo, that it is widely used in new high strength structural material manufacturing. The arc-shaped original bamboo pieces are mostly made of bamboo that is more than three years old as raw materials. It was sawn and processed into a curved blank with a length of 500 to $3000 \mathrm{~mm}$ and a chord length of 20 to $60 \mathrm{~mm}$. Through milling, a curved bamboo piece with smooth surface was formed. Arc-shaped original bamboo pieces can keep the shape of bamboo to the greatest extent, which is a new method to realize the efficient utilization of bamboo. The utilization rate of bamboo can reach more than $90 \%$, and it has high strength and can replace wood resources (Fei and Fu 2008; Zhou et al. 2016).

Zhang et al. (1997) used an intermittent temperature measurement method and a universal digital display meter to make statistical analysis on the core temperature of the arc-shaped original bamboo pieces, and obtained the variation rule of the bamboo pieces' temperature during high-frequency hot pressing. Many researchers (Avramidis et al. 1994; Zhang et al. 1997; Kawai et al. 2003; Nguyen et al. 2015; Chai et al. 2018) used a fiber optic temperature sensor to measure the core temperature and surface temperature of the wood during high-frequency heating, and they qualitatively analyzed the influence of moisture content on temperature. Huang et al. (2013) used fir as a test material and used intermittent heating to study the effect of wood moisture content, sample thickness, and RF (radio-frequency) power on heating rate. The results showed that the wood moisture content had a significant effect on the RF heating rate. Additionally, the thickness had no significant effect on the RF heating rate of the core layer, but the heating rate varied significantly in different parts along the thickness direction. The RF power level had a significant effect on the heating rate in the wood. The heating rate at low power level was initially higher than that at high power level, and then it decreased rapidly. During the whole heating process, the heating rate at high RF power level remained unchanged. However, due to the structural and physiological differences between wood and bamboo, the temperature variation law during high-frequency heating is also different (Huang et al. 2013).

The purpose of this study was to explore the heat transfer mechanism of the arcshaped original bamboo pieces in the high-frequency electric field, and analyze the effects of moisture content, chord length, and electrode plate voltage of high-frequency electric field on the heating rate of the arc-shaped original bamboo pieces. The results will better guide the post-forming process of the arc-shaped original bamboo pieces and broaden the application of high-frequency heating technology in the field of bamboo. Therefore, the purpose of this paper is also to explore the application potential of highfrequency heating technology in bamboo drying and gluing. From the perspective of bamboo utilization, the heat transfer mechanism of high-frequency electric field arcshaped bamboo is of great significance for the efficient use of bamboo.

\section{EXPERIMENTAL}

\section{Materials}

The test materials were bamboo from Yiyang City, Hunan Province, China, and the bamboo age was between 3 and 4 years. The water content of the raw materials was approximately $30 \%$, the length was $1200 \mathrm{~mm}$, the chord length was $40 \mathrm{~mm}$, and the thickness was $6 \mathrm{~mm}$. The production process of the arc-shaped original bamboo pieces are as follows. 
First, the original bamboo was cut into 1200-mm bamboo segments using a bamboo cutting machine (SZG-170-2000; Beijing Forestry Machinery Research Institute of State Forestry Administration, Beijing, China), and the bamboo segments were cut using a CNC (computer numerical control) automatic bamboo breaking machine (ASMG1; Beijing Forestry Machinery Research Institute of State Forestry Administration, Beijing, China). The corresponding number of segments were cut according to the diameter of the bamboo segments, so that the bamboo segments were cut into arc-shaped bamboo sheets with a chord length of approximately $40 \mathrm{~mm}$. After the initial drying, the bamboo inner skin and bamboo outer skin were removed by an arc milling machine (AFM-J1; Beijing Forestry Machinery Research Institute of State Forestry Administration, Beijing, China), and then the secondary milling was performed after adjusting the cutter and the clamping device. The width of the bamboo sheet was uniform, the radius of the arc was $50 \mathrm{~mm}$, the inner and outer parts of the bamboo were removed, and the surface was smooth (Zhou et al. 2015).

The test was divided into three groups (water content, chord length, and plate voltage). According to the width of high-frequency heating equipment, to make the arcshaped original bamboo pieces covered as much as possible by the electric field, bamboo raw materials were sawn into 490-mm-long bamboo segments. In the first group, the arcshaped original bamboo pieces were processed into samples of three chord lengths, which were the size of $20 \mathrm{~mm}, 30 \mathrm{~mm}$, and $40 \mathrm{~mm}$. In the second group, the moisture content was adjusted to $9 \%, 11 \%, 13 \%$, and $15 \%$. These samples were immediately wrapped with plastic film and kept in a cool room to prevent moisture loss. In the third group, all samples had the chord length of $40 \mathrm{~mm}$ for later use (Li et al. 2010). The dimensions and parameters of the arc-shaped original bamboo pieces used in the test are shown in Fig. 1 and Table 1.

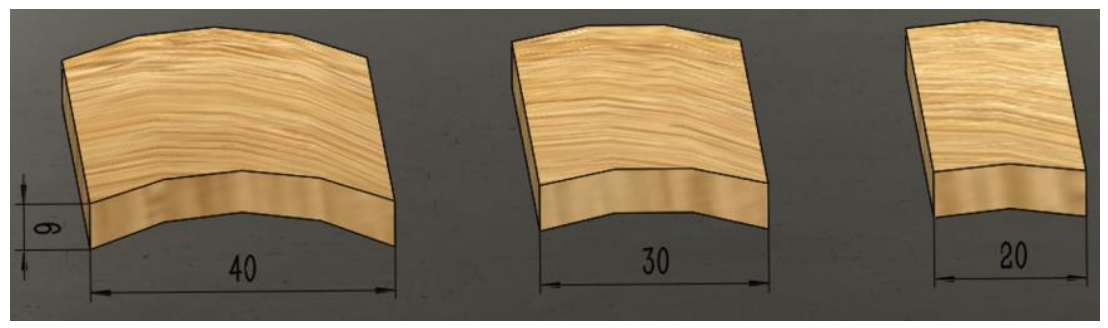

Fig. 1. The arc-shaped original bamboo pieces, all values are expressed in $\mathrm{mm}$

Table 1. Parameters of the Arc-shaped Original Bamboo Pieces for the Test

\begin{tabular}{|c|c|c|c|}
\hline Groups & Water Content (\%) & Chord Length $(\mathrm{mm})$ & Plate Voltage $(\mathrm{kV})$ \\
\hline \multirow{3}{*}{1} & 9 & 20 & 5 \\
\cline { 2 - 4 } & 9 & 30 & 5 \\
\cline { 2 - 4 } & 9 & 40 & 5 \\
\hline \multirow{3}{*}{2} & 9 & 40 & 5 \\
\cline { 2 - 4 } & 11 & 40 & 5 \\
\cline { 2 - 4 } & 13 & 40 & 5 \\
\hline \multirow{3}{*}{3} & 15 & 40 & 3 \\
\cline { 2 - 4 } & 11 & 40 & 5 \\
\cline { 2 - 4 } & 11 & 40 & 5.8 \\
\cline { 2 - 4 } & 11 & 40 & \\
\hline
\end{tabular}




\section{Methods}

The arc-shaped original bamboo pieces were heated by a GJ15-6B-1 (Shijiazhuang High-frequency Machinery Co., Ltd., Shijiangzhuang, China) highfrequency generator together with a GJB-PI-51B-JY (Shijiazhuang High-frequency Machinery Co., Ltd., Shijiangzhuang, China) high-frequency hydraulic press $(500 \mathrm{~mm} \times$ $300 \mathrm{~mm}$ in width). In the first group, the arc-shaped original bamboo pieces with different chord lengths were heated at a temperature of $50{ }^{\circ} \mathrm{C}$, and the heating times were compared for the different chord lengths. In the second group, the arc-shaped original bamboo pieces with different moisture contents were heated for 3 to $4 \mathrm{~min}$, and the heating rate and final temperature were observed. In the third group, arc bamboo pieces were heated under different electrode plate voltages to observe the heating condition. All tests were repeated three times.

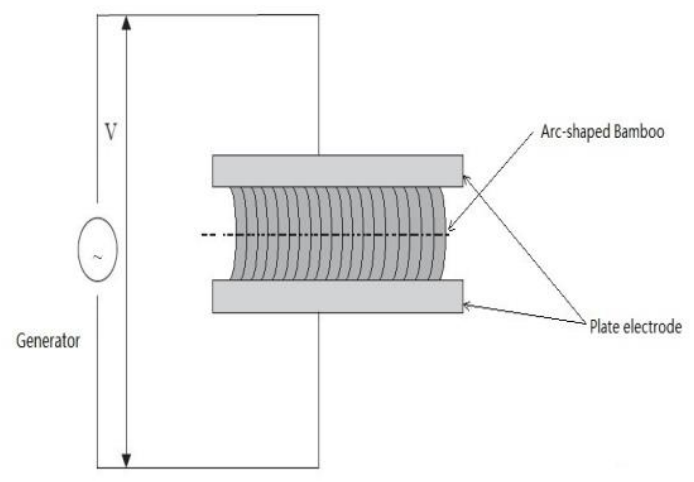

(a)

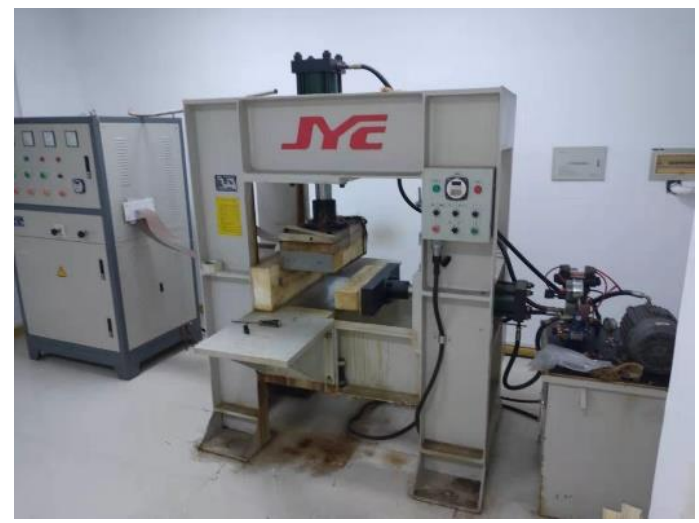

(b)

Fig. 2. (a) Schematic diagram and (b) equipment for heating arc-shaped bamboo pieces
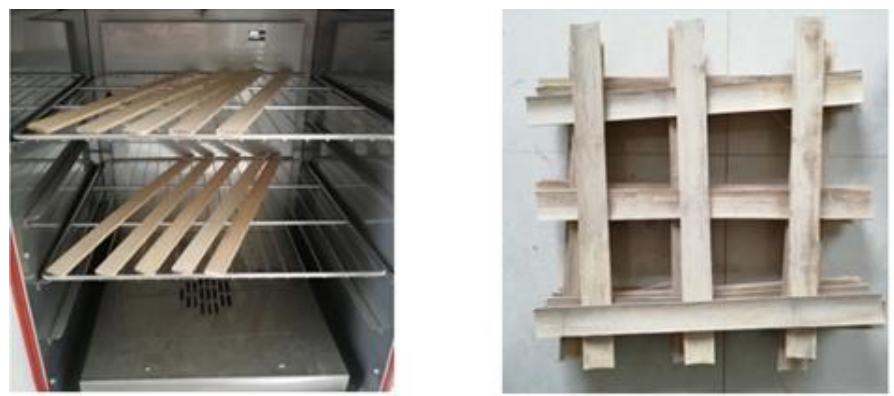

Adjust moisture content
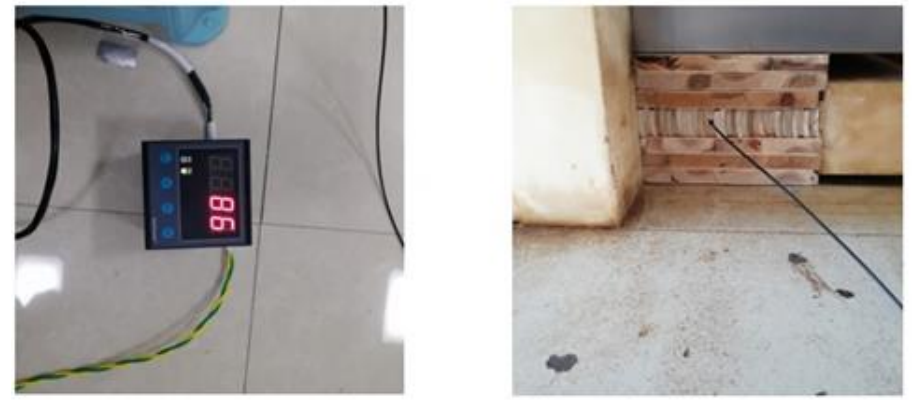

Paving and heating.

Fig. 3. Schematic diagram of arc-shaped bamboo pieces heating 


\section{Equations}

The intensity and distribution of electric field during heating can be expressed by the Maxwell equation (Alfaifi et al. 2014),

$$
-\nabla \cdot\left(\left(\sigma+j 2 \pi \varepsilon_{0} \varepsilon^{\prime}\right) \nabla V\right)=0
$$

where $\sigma$ is the conductivity of bamboo $(\mathrm{S} / \mathrm{m}), j=\sqrt{ }(-1), \varepsilon_{0}$ is the vacuum dielectric constant, $\varepsilon^{\prime}$ is the dielectric constant of bamboo, and $V$ is the plate voltage $(\mathrm{kV})$.

Heat conduction in high-frequency electric field is determined by the Fourier formula,

$$
\partial T / \partial t=\alpha \nabla^{2} T+P /\left(\rho c_{\mathrm{p}}\right)
$$

where $\partial T / \partial t$ is the instantaneous heating rate $\left({ }^{\circ} \mathrm{C} / \mathrm{S}\right)$ in the electric field, $\alpha$ is the thermal diffusion coefficient $\left(\mathrm{m}^{2} / \mathrm{s}\right), \rho$ is the density $\left(\mathrm{kg} / \mathrm{m}^{3}\right), c_{\mathrm{p}}$ is the specific heat $\left(\mathrm{j} / \mathrm{kg}^{\circ} \mathrm{C}\right)$, and $P$ is the power density $\left(\mathrm{W} / \mathrm{m}^{3}\right)$ of the unit volume stack.

The conversion of electromagnetic energy to thermal energy is expressed by the following equation (Choi and Konrad 1991),

$$
P=2 \pi \varepsilon_{0} \varepsilon^{\prime \prime}|E|^{2} \text { and } E=-\nabla V
$$

where $f$ is the operating frequency $(\mathrm{Hz})$ of high-frequency heating equipment, $\varepsilon^{\prime \prime}$ is the dielectric loss factor of bamboo, and $E$ is the electric field strength between polar plates.

\section{RESULTS}

\section{Effect of Chord Length on the Heating Rate of Arc-shaped Bamboo Pieces}

The conventional rectangular bamboo pieces heating did not need to take into account the chord length. It is necessary to consider the influence of chord length on the heating rate to study the factors affecting the heating rate of the arc-shaped bamboo. The chord length of the arc bamboo was different, the degree of its bending was different, and the thickness of heating in the electric field was also different. The shape of arc bamboo with a chord length of $20 \mathrm{~mm}$ was similar to that of conventional rectangular bamboo. However, for arc bamboo with a chord length of more than $30 \mathrm{~mm}$, its own curvature was large, and its heating mode in the electric field was quite different from that of rectangular bamboo. 


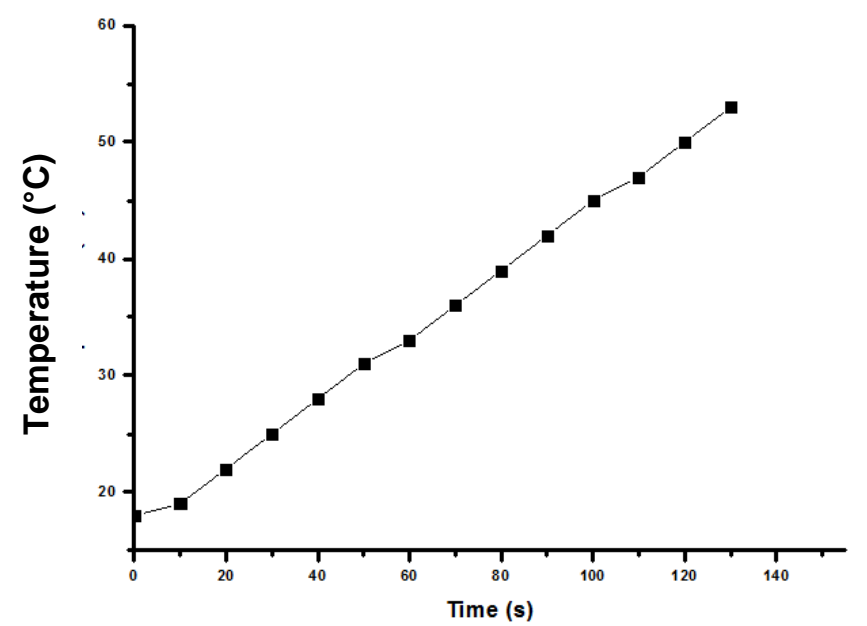

Time (s)

(a)

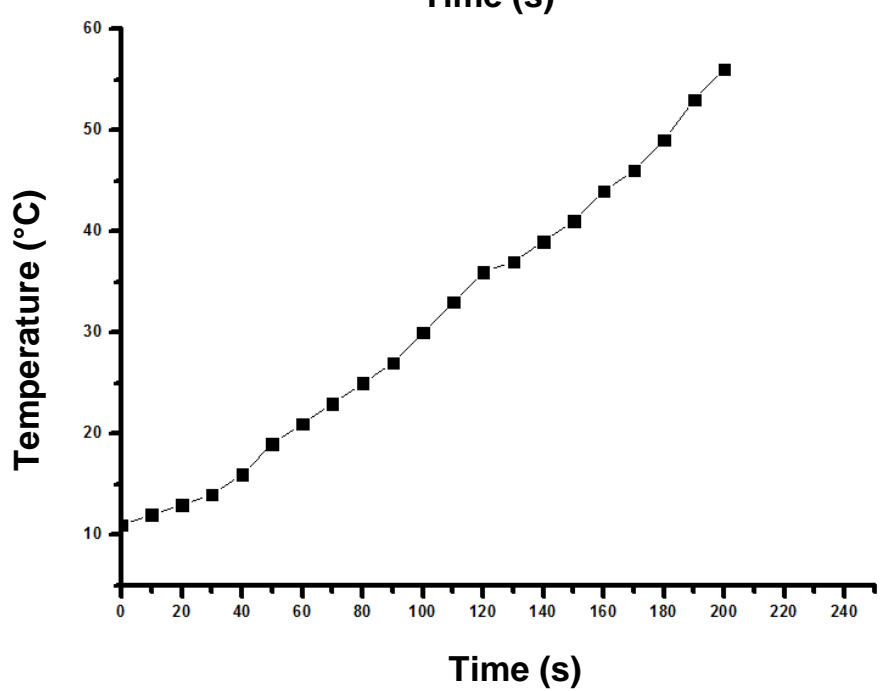

(b)

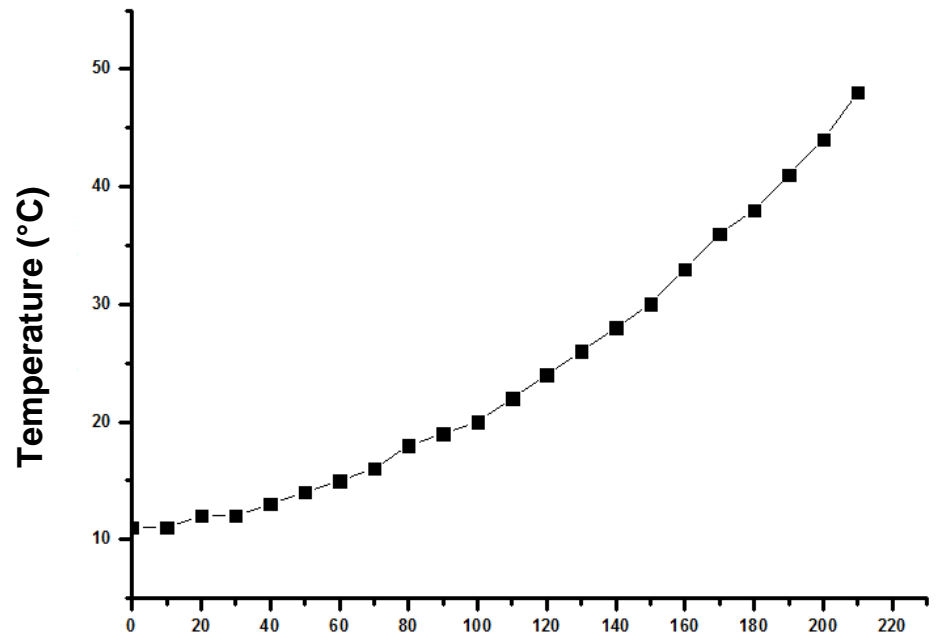

Time (s)

(c)

Fig. 4. Temperature-rising curves of different chord length arc-shaped bamboo pieces under high-frequency electric field: (a) $20 \mathrm{~mm}$, (b) $30 \mathrm{~mm}$, and (c) $40 \mathrm{~mm}$ 
According to Fig. 4, the heating rate of arc bamboo pieces with chord length of 20 $\mathrm{mm}$ was the fastest in high-frequency electric field, and the temperature reached $50{ }^{\circ} \mathrm{C}$ at $120 \mathrm{~s}$. At the same time, the temperatures of bamboo pieces with chord length of $30 \mathrm{~mm}$ and $40 \mathrm{~mm}$ were $36{ }^{\circ} \mathrm{C}$ and $24^{\circ} \mathrm{C}$, respectively. Moreover, according to Fig. 4(a), it was obvious that the heating rate curve of arc bamboo pieces with chord length of $20 \mathrm{~mm}$ was similar to that of conventional rectangular bamboo pieces. The chord length in Fig. 3(b) and Fig. 3(c) was not less than $30 \mathrm{~mm}$, and the heating rate in the high-frequency electric field was remarkably lower than that in the arc bamboo slice with the chord length of 20 $\mathrm{mm}$, as shown in Fig. 5.

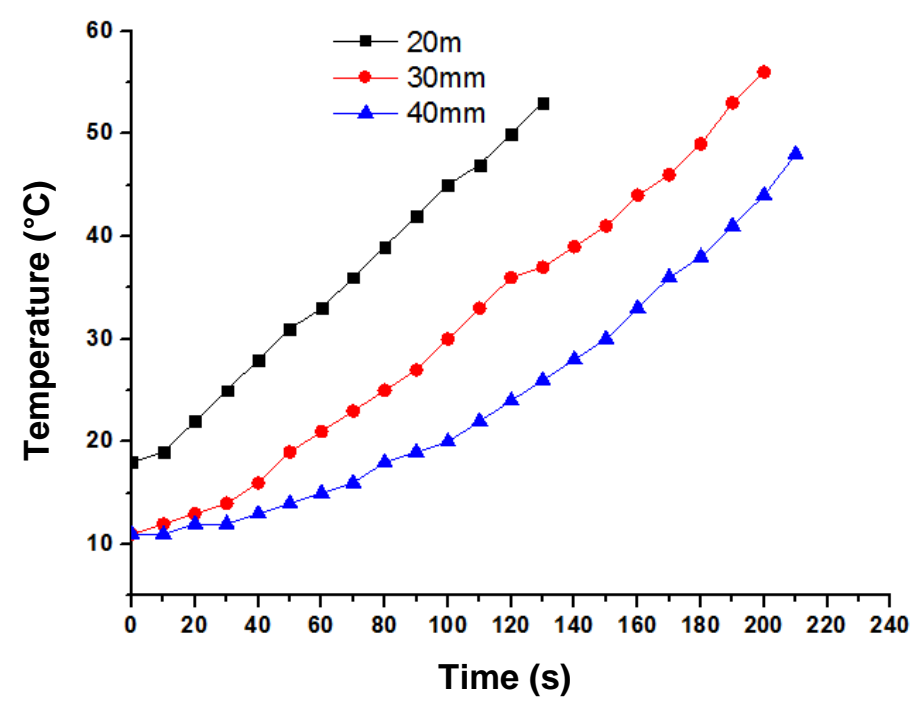

Fig. 5. Comparison of temperature-rising curves of bamboo pieces with different chord arc length under high-frequency electric field

It can be seen from Fig. 5 that the average heating rate of the curved bamboo pieces decreased as the chord length increased. The heating rate of the 20 -mm curved bamboo piece was higher than the heating rate of the curved bamboo piece of 30 to 40 $\mathrm{mm}$, and the heating rate of the chord length of $30 \mathrm{~mm}$ was also higher than the heating rate of the chord length of $40 \mathrm{~mm}$.

\section{Effect of Moisture Content on the Heating Rate of Arc Bamboo Pieces}

According to the conversion equation of electromagnetic energy to thermal energy, the electric field frequency $f(\mathrm{~Hz})$ and electric field strength $E$ were fixed in the test, so the main factor that affected the heating speed was the dielectric loss factor. A larger dielectric loss factor resulted in greater power density, greater amplitude, and greater frequency of polar molecule (such as water) movement resulted in greater amounts of heat generated by friction, and faster heating speed (Chen et al. 2007; Zhu and Guo 2010). 


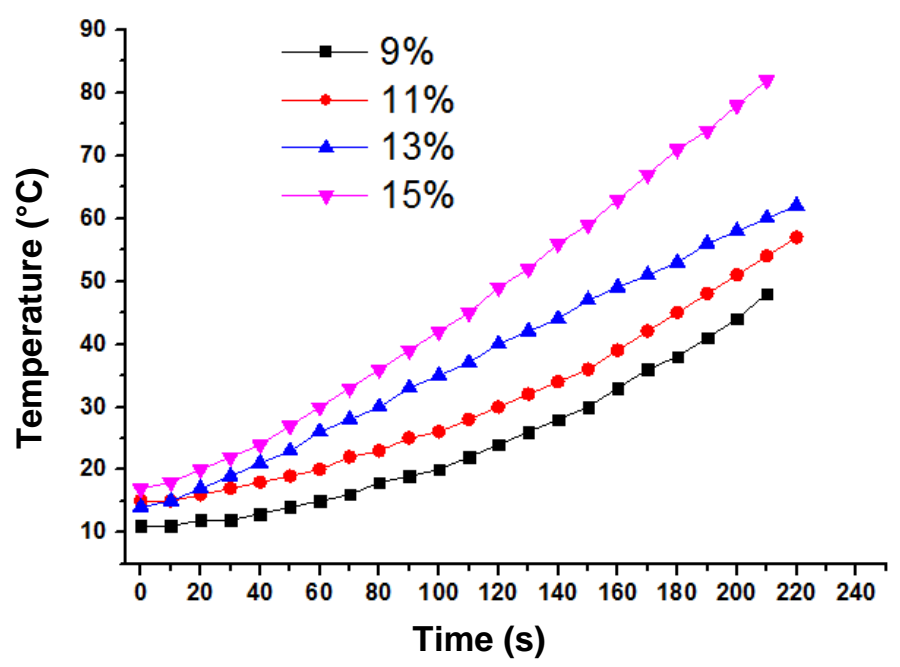

Fig. 6. Heating curves of arc bamboo pieces with different moisture contents under highfrequency electric field

However, the dielectric loss factor was closely related to the initial moisture content of the arc-shaped bamboo pieces. In the process of high-frequency heating, a higher moisture content of bamboo pieces resulted in more internal moisture content and a higher dielectric constant.

In the test, the results showed that with the increase of water content, the average rate of temperature rise accelerated. A higher water content resulted in a faster temperature increase at the same time, and the average rate of temperature rise accelerated with the increase of water content. When the moisture content of arc bamboo pieces was $9 \%$, it took $220 \mathrm{~s}$ to reach $50{ }^{\circ} \mathrm{C}$. When the moisture content of arc bamboo pieces was $15 \%$, it needed $130 \mathrm{~s}$ to reach $50{ }^{\circ} \mathrm{C}$, as shown in Fig. 6. However, according to the temperature-rise curve, with increased water content, the growth of instantaneous temperature rise rate tended to slow down, and the acceleration of the temperature rise curve became smaller.

\section{Effect of Pole Plate Voltage on Heating Rate of Arc-shaped Bamboo Pieces}

According to the heating principle of arc bamboo pieces in high-frequency electric field, the magnitude of electrode plate voltage determines the strength of electric field. In general heating conditions, the higher the electrode plate voltage, the stronger the high-frequency electric field strength, and the faster the heating rate. However, due to the limitation of the width of the high-frequency heating platform used in the test, the test set the voltage of the plate in the 4th gear and selected the appropriate voltage gear according to the test results. The test results are shown in Fig. 7. 


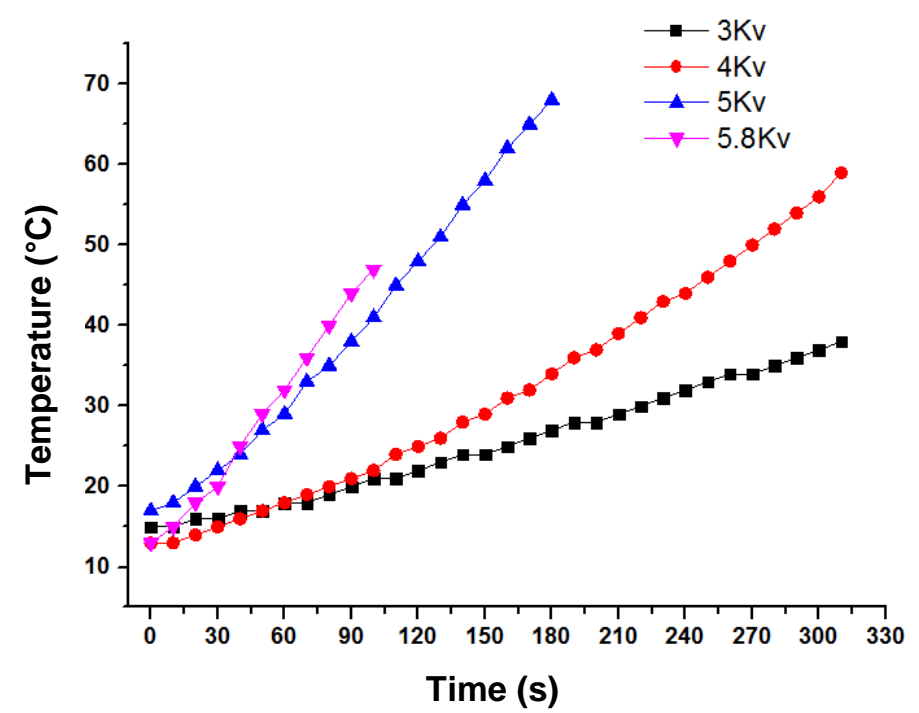

Fig. 7. Temperature-rise curves of arc bamboo pieces in high-frequency electric field under different electrode plate voltages

According to the test results, the average heating rate of the curved bamboo in the high-frequency electric field increased remarkably with the increase of the plate voltage, especially in the gear shifting of $4 \mathrm{kV}$ to $5 \mathrm{kV}$, the heating rate of the curved bamboo was particularly increased. When the voltage was $3 \mathrm{kV}$, the heating rate of arc bamboo pieces was slow, the heating time reached $300 \mathrm{~s}$, and the temperature of arc bamboo pieces was only $37{ }^{\circ} \mathrm{C}$. When the voltage was $4 \mathrm{kV}$, the heating rate of bamboo pieces rose steadily, the heating curve was smooth, and the heating rate was faster. When the voltage was 5 $\mathrm{kV}$, the temperature rise rate of arc bamboo pieces increased rapidly and the temperature rose rapidly. However, the high-frequency electric field occasionally broke down when heated at the voltage of $5 \mathrm{kV}$ plate. At $5.8 \mathrm{kV}$, the temperature of the arc-shaped bamboo chip rose rapidly in the high-frequency electric field, but it was extremely unstable. The whole electric field broke down and the heating was interrupted after heating for $1 \mathrm{~min}$. The heating curve of gear $5.8 \mathrm{kV}$ is shown in Fig. 8.

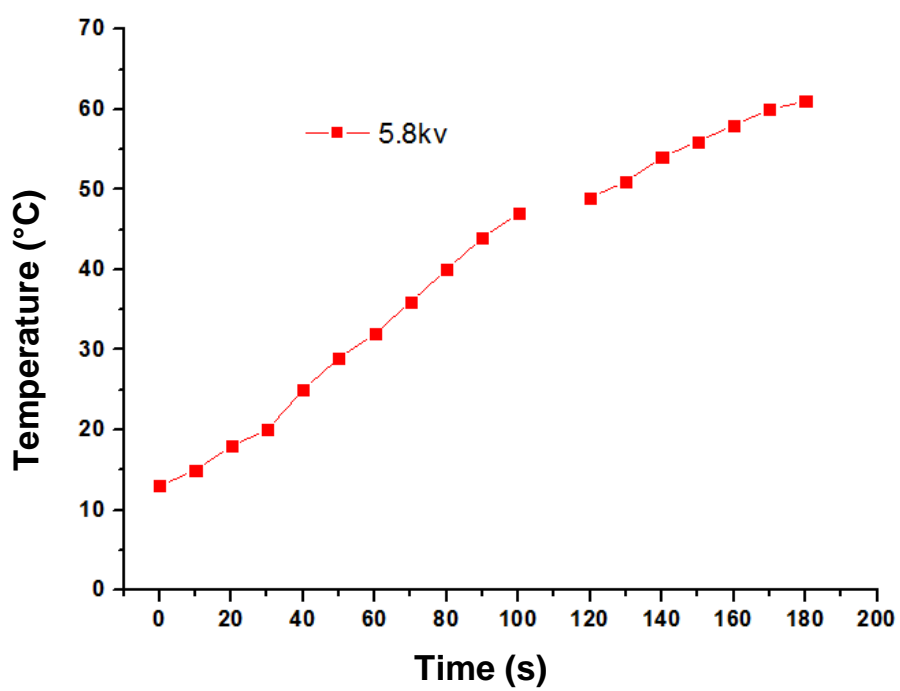

Fig. 8. Temperature-rise rate curve of arc bamboo under $5.8 \mathrm{kV}$ plate voltage 
It can be seen from Fig. 7 that when the pole plate voltage was $5.8 \mathrm{kV}$ and the arcshaped bamboo piece was heated for approximately $100 \mathrm{~s}$ in the high-frequency electric field, electric field breakdown occurred, which led to heating interruption. However, the temperature measured by the optical fiber temperature sensor after the interruption of heating slowly increased, and finally the temperature was up to $61{ }^{\circ} \mathrm{C}$. From the results of this test, it can be preliminarily concluded that the selection of heating plate voltage of arc bamboo pieces under high-frequency electric field should not be too large or too small.

\section{DISCUSSION}

The purpose of this study was to explore the factors that affect the temperature rise of arc-shaped bamboo pieces in high-frequency electric field, further explore the heating mechanism of arc-shaped bamboo pieces in high-frequency electric field, and better explain the influence on the quality performance of the subsequent bamboo molding. Nguyen et al. (2014) verified the temperature change trend of the slab during high-frequency hot pressing with different moisture contents of bamboo-integrated materials, and concluded that the heating effect of the slab was the best at the moisture content of $12 \%$. According to the analysis of the results in this experiment, the heating rate was the fastest when the moisture content was $15 \%$, but considering the utilization of bamboo and the stability of heating, curved bamboo pieces with a moisture content of approximately $11 \%$ had a stable heating curve, and the moisture content range was suitable for the forming and utilization of bamboo in the later stage.

The influence of the chord length of the curved bamboo on the heating rate was noticeable. When the chord length was greater than $30 \mathrm{~mm}$, the heating rate was obviously slowed down. At present, there is no research on the influence of the curved original bamboo structure on the heating temperature. It was concluded that when the chord length was more than $30 \mathrm{~mm}$, the bending degree of the curved bamboo was obvious, which may have hindered the reciprocating movement of polar water molecules. Because in the high-frequency alternating electric field, the temperature change of the dielectric material is generated by the friction of polar molecules moving rapidly along the direction of the electric field. When the bending is too large, it presumably has a blocking effect on the movement of polar molecules. The micro factors of the influence of the chord length of the arc-shaped original bamboo pieces on the high frequency heating need to be further studied.

The plate voltage determines the electric field strength. According to the relevant theory, the thermal power absorbed by the bamboo in the high-frequency electric field can be expressed by the power density $P\left(\mathrm{~W} \cdot \mathrm{cm}^{-3}\right), P=2 \pi f \varepsilon_{0} \varepsilon^{\prime \prime}|E|^{2}$, where the electric field strength $E=U / d$ is proportional to the plate voltage $U(\mathrm{kV})$ and inversely proportional to the thickness $d(\mathrm{~cm})$ between the plates. According to the formula, the stronger the electric field strength is, the faster the temperature rises. However, due to the limited height of the arc-shaped bamboo sheet and its large water content, when the electric field strength was too strong on the spot, the electric field breakdown easily occurred, resulting in the interruption of heating. Therefore, according to the test results, the voltage selection of arc bamboo heating plate should refer to the test plate voltage and plate thickness. 


\section{CONCLUSIONS}

1. Based on the study of the heating mechanism of the arc-shaped original bamboo pieces in the high-frequency electric field, the experiment was conducted by a single variable method. Under certain controllable conditions, the arc-shaped original bamboo pieces exhibited a better heat treatment effect in the high-frequency electric field.

2. The heat transfer mechanism showed no obvious quantitative relation. However, according to the present experimental results, the qualitative relation has been summarized.

3. In the heat treatment process of the arc-shaped original bamboo pieces using highfrequency electric field, the moisture content should not be too high. A moisture content below approximately $11 \%$ is recommended, thus a stable heating effect and being beneficial to the forming and utilization of bamboo pieces in the later stage. The selection of chord length of the arc-shaped original bamboo pieces should not only be conducive to stable heat treatment effect, but also realize efficient utilization of bamboo. Therefore, the selection of chord length should not be too large, i.e. in the range between 20 to $30 \mathrm{~mm}$. The plate voltage in the high-frequency heat treatment of the arc-shaped original bamboo pieces can be completed with reference to the $4 \mathrm{kV}$ gear position of the test.

\section{ACKNOWLEDGMENTS}

The authors are grateful for the financial support from the Basic Research Fund of the Chinese Academy of Forestry (Grant No. CAFYBB2017QB008) and the National Natural Science Foundation of China (NSFC; Grant No. 31500481).

\section{REFERENCES CITED}

Alfaifi, B., Tang, J., and Jiao, Y. (2014). "Radio frequency disinfestation treatments for dried fruit: Model development and validation," Journal of Food Engineering 120(1), 268-276. DOI: 10.1016/j.jfoodeng.2013.07.015

Avramidis, S., Liu, F., and Neilson, B. J. (1994). "Radio-frequency/vacuum drying of softwoods: Drying of thick western redcedar with constant electrode voltage," Forest Products Journal 44, 41-47.

Chai, H. J., Zhao, J. Y., and Cai, Y. C. (2018). "Development and validation of a simulation model for the temperature field during high-frequency heating of wood," Forests 9(6), Article Number 327. DOI: 10.3390/f9060327

Chang, F. H., Zhang, B., Fu, W. S., Zhang, F., and Zhou, J. B. (2018). "Application and research of high frequency technology in bamboo processing equipment," Wood Processing Machinery 29(5), 34-36, +4. DOI: 10.13594/j.cnki.mcjgjx.2018.05.007

Chen, Y. P., Wang, J. L., Li, C. S., and Wang, Z. T. (2007). "The application of highfrequency heating technology in wood bonding process," Wood Processing Machinery 18(5), 37-41. DOI: 10.3969/j.issn.1001-036X.2007.05.011 
Choi, C. T. M., and Konrad, A. (1991). "Finite element modeling of the RF heating process," IEEE Transaction and Magnetics 27(5), 4227-4230. DOI: $10.1109 / 20.105034$

Fan, X. P. (2014). "Analysis of development prospect for wooden structure building in China," Sichuan Building Materials 40(6), 91-92.

Fei, B., and Fu, W. (2008). "The arc bamboo original state of recombinant material and the manufacturing method," China Patent No. 200610114353.

Guo, W., Fei, B. H., and Chen, E. L. (2009). "Wood structural construction industry in China," China Wood Industry 23(2), 19-22.

Huang, R. F., Wu, Y. M., Zhao, Y. K., Lu, J. X., Jiang, J. L., and Chen, Z. J. (2013). "Factors affecting the temperature increasing rate in wood during radio-frequency heating," Drying Technology 31(2), 246-252. DOI: 10.1080/07373937.2012.728269

Kawai, Y., Kobayashi, Y., and Ishimaru, Y. (2003). "Hybrid drying with high frequency heating and hot-air under atmospheric pressure V: Combined effect of high frequency heating and hot air," Wood Research Society 49, 408-415.

Li, W., Zhang, Z. K., and Li, W. G. (2010). "Change in radius of moso arc spatulate during drying," Journal of Bamboo Research 29(1), 45-49. DOI: 10.3969/j.issn.1000-6567.2010.01.010

Lv, Y. Y., Fu, Z. Y., and Song, T. Y. (2015). "The effect of radio-frequent heating on water migration for boxed-heart larch lumber," Journal of Anhui Agriculture Science 2(9), 148-151. DOI: 10.13989/j.cnki.0517-6611.2015.09.054

Nguyen, T. H. G., and Zhang, Q. S. (2015). "Temperature inside mats of high-frequency, hot pressed, glued and laminated bamboo," Journal of Zhejiang Agriculture and Forest University 32(2), 167-172. DOI: 10.11833/j.issn.2095-0756.2015.02.001

Pereira, M. C., Blanchard, C., and Carvalho, L. M. H. (2004). "High frequency heating of medium density fiberboard (MDF): Theory and experiment," Chemical Engineering Science 59(4), 735-745. DOI: 10.1016/j.ces.2003.09.038

Zhang, L., Avramidis, S., and Hatzikiriakos, S. G. (1997). "Moisture flow characteristics during radio frequency vacuum drying of thick lumber," Wood Science and Technology 31, 265-277. DOI: 10.1007/BF00702614

Zhou, J. B., Chen, S. L., Fu, W. S., Chen, Z. J., Zhao, Z. R., Cheng, W. L., and Zhang, Z. K. (2016). "Preparation and mechanic property evaluation of bamboo lumber prepared by assembly and glue-curing of naturally arc-shaped segments with finger joints," BioResources 11(1), 267-280. DOI: 10.15376/biores.11.1.267-280

Zhou, J. B., Fu, W. S., Qing, Y., Zhao, Z. R., and Zhang, B. (2015). "Fabrication and performance of a glue-pressed engineered honeycomb bamboo (GPEHB) structure with finger-jointed ends as a potential substitute for wood lumber," BioResources 10(2), 3302-3313. DOI: 10.15376/biores.10.2.3302-3313

Zhu, X. H., and Guo, W. C. (2010). "A review of affecting factors and their mechanisms of the radio frequency-microwave dielectric properties of foods," Food Science 31(17), 410-414.

Article submitted: December 5, 2019; Peer review completed: February 13, 2020; Revised version received: February 22, 2020; Published: February 25, 2020.

DOI: 10.15376/biores.15.2.2656-2667 